$w$-SEMIOPEN SETS

\title{
AND $w$-SEMICONTINUITY IN WEAK SPACES
}

\author{
Won Keun Min ${ }^{1 \S}$, Young Key Kim ${ }^{2}$ \\ ${ }^{1}$ Department of Mathematics \\ Kangwon National University \\ Chuncheon, 200-701, KOREA \\ ${ }^{2}$ Department of Mathematics \\ MyongJi University \\ Yongin, 449-728, KOREA
}

\begin{abstract}
The purpose of this short note is to generalize $w$-open sets in $w$-spaces, say $w$-semiopen sets. First, we introduce the notion of $w$-semiopen sets and some basic properties of such the notion. Second, we introduce $W$-semicontinuous functions defined by $w$-semiopen sets and investigate characterizations of them by using the several types of operators.
\end{abstract}

AMS Subject Classification: 54A05, 54B10, 54C10

Key Words: $w$-spaces, $w$-open, $W$-continuous, $w$-semiopen, $W$-semicontinuous.

\section{Introduction}

Siwiec [16] introduced the notions of weak neighborhoods and weak base in a topological space. We introduced the weak neighborhood systems defined by using the notion of weak neighborhoods in [13]. The weak neighborhood system induces a weak neighborhood space which is independent of neighborhood spaces [4] and general topological spaces [2]. The notions of weak structure, $w$ space, $W$-continuity and $W^{*}$-continuity were investigated in [14]. In fact, the set of all $g$-closed subsets [5] in a topological space is a kind of weak structure.

\footnotetext{
Received: June 9, 2016

Revised: July 20, 20166

Published: October 21, 2016

(c) 2016 Academic Publications, Ltd. url: www.acadpubl.eu

$\S_{\text {Correspondence author }}$
} 
The one purpose of our research is to generalize $w$-open sets in $w$-spaces. In 1963, Levine [6] introduced the concept of semi-open set in topological spaces and used this to define other new concepts such as semi-closed set and semicontinuity of a function. In this paper, in the same way as Levine did, we are going to study the notion of $w$-semiopen set in a weak space.

First, we investigate the notion of $w$-semiopen sets and some basic properties of such the notion. Second, we introduce $W$-semicontinuous functions defined by $w$-semiopen sets, and study characterizations of them by using the operators $w C, w I, w s C$ and $w s I$.

\section{Preliminaries}

Definition 1 ([14]). Let $X$ be a nonempty set. A subfamily $w_{X}$ of the power set $P(X)$ is called a weak structure on $X$ if it satisfies the following:

(1) $\emptyset \in w_{X}$ and $X \in w_{X}$.

(2) For $U_{1}, U_{2} \in w_{X}, U_{1} \cap U_{2} \in w_{X}$.

Then the pair $\left(X, w_{X}\right)$ is called a $w$-space on $X$. Then $V \in w_{X}$ is called a $w$-open set and the complement of a $w$-open set is a $w$-closed set.

The collection of all $w$-open sets (resp., $w$-closed sets) in a $w$-space $X$ will be denoted by $W O(X)$ (resp., $W C(X))$. We set $W(x)=\{U \in W O(X): x \in U\}$.

Let $S$ be a subset of a topological space $X$. The closure (resp., interior) of $S$ will be denoted by $c l S$ (resp., int $S$ ). A subset $S$ of $X$ is called a preopen set [10] (resp., $\alpha$-open set [15], semi-open [6]) if $S \subset \operatorname{int}(\operatorname{cl}(S))$ (resp., $S \subset$ $\operatorname{int}(\operatorname{cl}(\operatorname{int}(S))), S \subset \operatorname{cl}(\operatorname{int}(S)))$. The complement of a preopen set (resp., $\alpha$ open set, semi-open) is called a preclosed set (resp., $\alpha$-closed set, semi-closed). The family of all preopen sets (resp., $\alpha$-open sets, semi-open sets) in $X$ will be denoted by $P O(X)$ (resp., $\alpha(X), S O(X)$ ). We know the family $\alpha(X)$ is a topology finer than the given topology on $X$.

A subset $A$ of a topological space $(X, \tau)$ is said to be:

(a) $g$-closed [5] if $\operatorname{cl}(A) \subset U$ whenever $A \subset U$ and $U$ is open in $X$;

(b) $g p$-closed [7] if $p C l(A) \subset U$ whenever $A \subset U$ and $U$ is open in $X$;

(c) $g s$-closed $[1,3]$ if $s C l(A) \subset U$ whenever $A \subset U$ and $U$ is open in $X$;

(d) $g \alpha$-closed [9] if $\tau^{\alpha} C l(A) \subset U$ whenever $A \subset U$ and $U$ is $\alpha$-open in $X$ where $\tau^{\alpha}=\alpha(X)$; $X$;

(e) $g \alpha^{*}$-closed [8] if $\tau^{\alpha} C l(A) \subset \operatorname{int}(U)$ whenever $A \subset U$ and $U$ is $\alpha$-open in

(f) $g \alpha^{* *}$-closed [8] if $\tau^{\alpha} C l(A) \subset \operatorname{int}(\operatorname{cl}(U))$ whenever $A \subset U$ and $U$ is $\alpha$-open in $X$; 
(g) $\alpha g$-closed [9] if $\tau^{\alpha} C l(A) \subset U$ whenever $A \subset U$ and $U$ is open in $X$; in $X$.

(h) $\alpha^{* *} g$-closed [9] if $\tau^{\alpha} C l(A) \subset \operatorname{int}(\operatorname{cl}(U))$ whenever $A \subset U$ and $U$ is open

Then the family $\tau, G O(X), g \alpha O(X), g \alpha^{*} O(X), g \alpha^{* *} O(X), \alpha g O(X)$ and $\alpha^{* *} g O(X)$ are all weak structures on $X$. But $P O(X), G P O(X)$ and $S O(X)$ are not weak structures on $X$. A subfamily $m_{X}$ of the power set $P(X)$ of a nonempty set $X$ is called a minimal structure on $X[10]$ if $\emptyset \in w_{X}$ and $X \in w_{X}$. Thus clearly every weak structure is a minimal structure.

Definition $2([14])$. Let $\left(X, w_{X}\right)$ be a $w$-space. For a subset $A$ of $X$, the $w$-closure of $A$ and the w-interior of $A$ are defined as follows:

(1) $w C(A)=\cap\left\{F: A \subseteq F, X-F \in w_{X}\right\}$.

(2) $w I(A)=\cup\left\{U: U \subseteq A, U \in w_{X}\right\}$.

Theorem 3 ([14]). Let $\left(X, w_{X}\right)$ be a $w$-space and $A \subseteq X$.

(1) $x \in w I(A)$ if and only if there exists an element $U \in W(x)$ such that $U \subseteq A$.

(2) $x \in w C(A)$ if and only if $A \cap V \neq \emptyset$ for all $V \in W(x)$.

(3) If $A \subset B$, then $w I(A) \subset w I(B)$; $w C(A) \subset w C(B)$.

(4) $w C(X-A)=X-w I(A) ; w I(X-A)=X-w C(A)$.

(5) If $A$ is $w$-closed (resp., w-open), then $w C(A)=A$ (resp., $w I(A)=A$ ).

Definition 4 ([14]). Let $f: X \rightarrow Y$ be a function on $w$-spaces $\left(X, w_{X}\right)$ and $\left(Y, w_{Y}\right)$. Then $f$ is said to be $W$-continuous [14] if for $x \in X$ and $V \in W(f(x))$, there is $U \in W(x)$ such that $f(U) \subseteq V$.

\section{3. $w$-Semiopen Sets, $W$-Semicontinuity}

Definition 5. Let $\left(X, w_{X}\right)$ be a $w$-space and $S \subseteq X$. Then $S$ is called a $w$-semiopen set if $S \subseteq w C(w I(S))$. The complement of a $w$-semiopen set is called a $w$-semiclosed set.

The family of all $w$-semiopen sets in $X$ will be denoted by $W S O(X)$.

Lemma 6. Let $\left(X, w_{X}\right)$ be a $w$-space and $A \subseteq X$. Then the following things hold:

(1) $A$ is a $w$-semiclosed set if and only if $w I(w C(A)) \subseteq A$.

(2) Every w-open (resp., w-closed) set is $w$-semiopen (resp., w-semiclosed).

We can easily show that a $w$-semiopen set may not be $w$-open as the next example: 
Example 7. Let $X=\{a, b, c\}$ and $w_{X}=\{\emptyset,\{a\},\{b\}, X\}$ be a $w$-structure in $X$. Consider $A=\{a, b\}$; then $w C(w I(A))=X$. So $A$ is $w$-semiopen but not $w$-open.

Theorem 8. Let $\left(X, w_{X}\right)$ be a $w$-space. Any union of $w$-semiopen sets is also $w$-semiopen.

Proof. Let $A_{i}$ be a $w$-semiopen set for $i \in J$. From (3) of Theorem 2.3, $A_{i} \subseteq w C\left(w I\left(A_{i}\right)\right) \subseteq w C\left(w I\left(\cup A_{i}\right)\right)$. Clearly, this implies $\cup A_{i} \subseteq w C\left(w I\left(\cup A_{i}\right)\right)$ and so $\cup A_{i}$ is $w$-semiopen.

In general, the intersection of any two $w$-semiopen sets is not $w$-semiopen. See the next example:

Example 9. In example 3.3, consider $A=\{a, c\}$ and $B=\{b, c\}$. Note that $w C(w I(A))=A$ and $w C(w I(B))=B$. So both $A$ and $B$ are $w$-semiopen, but $A \cap B=\{c\}$ is not $w$-semiopen.

Definition 10. Let $\left(X, w_{X}\right)$ be a $w$-space and $A \subseteq X$. Then the $w$-semiclosure of $A$ and the w-semi-interior of $A$, denoted by $w s C(A)$ and $w s I(A)$, respectively, are defined as:

$$
\begin{gathered}
w s C(A)=\cap\{F: A \subseteq F, F \text { is } w \text {-semiclosed in } X\} ; \\
w s I(A)=\cup\{U: U \subseteq A, U \text { is } w \text {-semiopen in } X\} .
\end{gathered}
$$

Theorem 11. Let $\left(X, w_{X}\right)$ be a $w$-space and $A, B, F \subseteq X$. Then the following things hold:

(1) $w s I(A) \subseteq A ; A \subseteq w s C(A)$.

(2) If $A \subseteq B$, then $w s I(A) \subseteq w s I(B)$ and $w s C(A) \subseteq w s C(B)$.

(3) $A$ is $w$-semiopen iff $w s I(A)=A$.

(4) $F$ is $w$-semiclosed iff $w s C(F)=F$.

(5) $w s I(w s I(A))=w s I(A)$; wsC $(w s C(A))=w s C(A)$.

(6) $w s C(X-A)=X-w s I(A)$; wsI $(X-A)=X-w s C(A)$.

Proof. (1) and (2) Obvious.

(3) and (4) It follows from Theorem 3.5.

(5) It follows from (3) and (4).

(6) For $A \subseteq X$,

$$
\begin{aligned}
X-w s I(A) & =X-\cup\{U: U \subseteq A, U \text { is } w \text {-semiopen }\} \\
& =\cap\{X-U: U \subseteq A, U \text { is } w \text {-semiopen }\} \\
& =\cap\{X-U: X-A \subseteq X-U, U \text { is } w \text {-semiopen }\} \\
& =w s C(X-A) .
\end{aligned}
$$


Similarly, we have $w s I(X-A)=X-w s C(A)$.

Theorem 12. Let $\left(X, w_{X}\right)$ be a $w$-space and $A \subseteq X$. Then

(1) $x \in w s C(A)$ if and only if $A \cap V \neq \emptyset$ for every $w$-semiopen set $V$ containing $x$.

(2) $x \in w s I(A)$ if and only if there exists a $w$-semiopen set $U$ such that $U \subseteq A$.

Proof. (1) Suppose that there is a $w$-semiopen set $V$ containing $x$ such that $A \cap V=\emptyset$. Then $X-V$ is a $w$-semiclosed set such that $A \subseteq X-V$ and $x \notin X-V$. This implies $x \notin w s C(A)$.

The reverse relation is obvious.

(2) Obvious.

Now, we introduce the notion of $W$-semicontinuous functions defined by $w$-semiopen sets, and study characterizations of them by using the operators $w C, w I, w s C$ and $w s I$.

Definition 13. Let $f:\left(X, w_{X}\right) \rightarrow\left(Y, w_{Y}\right)$ be a function on $w$-spaces $X$ and $Y$. Then $f$ is said to be $W$-semicontinuous if for each $x$ and each $w$-open set $V$ containing $f(x)$, there exists a $w$-semiopen set $U$ containing $x$ such that $f(U) \subseteq V$.

Since every $w$-open set is $w$-semiopen, obviously, a $W$-continuous function is $W$-semicontinuous, but the converse is not true in general.

$$
W \text {-continuity } \Rightarrow W \text {-semicontinuity }
$$

Example 14. Let $X=\{a, b, c, d\}$. Consider two weak structures $w_{1}$ and $w_{2}$, respectively, as the following:

$w_{1}=\{\emptyset,\{a\},\{b\},\{a, b\}, X\} ; w_{2}=\{\emptyset,\{a, b\},\{a\},\{a, c\}, X\}$.

Let $f:\left(X, w_{1}\right) \rightarrow\left(X, w_{2}\right)$ be the identity function. Then $f$ is $W$-semicontinuous but not $W$-continuous.

Theorem 15. Let $f:\left(X, w_{X}\right) \rightarrow\left(Y, w_{Y}\right)$ be a function on $w$-spaces $X$ and $Y$. Then the following statements are equivalent:

(1) $f$ is $W$-semicontinuous.

(2) For each $w$-open set $V$ in $Y, f^{-1}(V)$ is $w$-semiopen.

(3) For each $w$-closed set $B$ in $Y, f^{-1}(B)$ is $w$-semiclosed.

(4) $f(w s C(A)) \subseteq w C(f(A))$ for $A \subseteq X$.

(5) $w s C\left(f^{-1}(B)\right) \subseteq f^{-1}(w C(B))$ for $B \subseteq Y$.

(6) $f^{-1}(w I(B)) \subseteq w s I\left(f^{-1}(B)\right)$ for $B \subseteq Y$. 
Proof. $(1) \Rightarrow(2)$ Let $V$ be a $w$-open set in $Y$. Then for each $x \in f^{-1}(V)$, by the hypothesis, there exists a $w$-semiopen set $U_{x}$ containing $x$ such that $f(U) \subseteq V$. It implies that for each $x \in f^{-1}(V), x \in U_{x} \subseteq f^{-1}(V)$. Hence by Theorem 3.4, $f^{-1}(V)$ is $w$-semiopen.

$(2) \Rightarrow(3)$ Obvious.

$(3) \Rightarrow(4)$ For $A \subseteq X$,

$$
\begin{aligned}
f^{-1}( & w(f(A))) \\
& =f^{-1}(\cap\{F \subseteq Y: f(A) \subseteq F \text { and } F \text { is } w \text {-closed }\}) \\
& =\cap\left\{f^{-1}(F) \subseteq X: A \subseteq f^{-1}(F) \text { and } f^{-1}(F) \text { is } w \text {-semiclosed }\right\} \\
& \supseteq \cap\{K \subseteq X: A \subseteq K \text { and } K \text { is } w \text {-semiclosed }\} \\
& =w s C(A)
\end{aligned}
$$

It implies that $f(w s C(A)) \subseteq w C(f(A))$.

(4) $\Rightarrow(5)$ For $B \subseteq Y$, from (4), it follows

$$
f\left(w s C\left(f^{-1}(B)\right)\right) \subseteq w C\left(f\left(f^{-1}(B)\right)\right) \subseteq w C(B)
$$

So, $w s C\left(f^{-1}(B)\right) \subseteq f^{-1}(w C(B))$.

$(5) \Rightarrow(6)$ For $B \subseteq Y$, from $w I(B)=Y-w C(Y-B)$ and (5), it follows

$$
\begin{aligned}
f^{-1}(w I(B)) & =f^{-1}(Y-w C(Y-B)) \\
& =X-\left(f^{-1}(w C(Y-B))\right) \\
& \subseteq X-w s C\left(f^{-1}(Y-B)\right) \\
& =w s I\left(f^{-1}(B)\right) .
\end{aligned}
$$

So, $f^{-1}(w I(B)) \subseteq w s I\left(f^{-1}(B)\right)$.

$(6) \Rightarrow(1)$ Let $x \in X$ and $V$ be a $w$-open set containing $f(x)$. Then, by Theorem 2.3 and (6), $x \in f^{-1}(V)=f^{-1}(w I(V)) \subseteq w s I\left(f^{-1}(V)\right)$. So, there exists a $w$-semiopen set $U$ containing $x$ such that $x \in U \subseteq f^{-1}(V)$. It implies that $f$ is $W$-semicontinuous.

Lemma 16. Let $\left(X, w_{X}\right)$ be a $w$-space and $A \subseteq X$. Then

(1) $w I(w C(A)) \subseteq w I(w C(w s C(A))) \subseteq w s C(A)$.

(2) $w s I(A) \subseteq w C l(w I(w s I(A))) \subseteq w I(w C(A))$. 
Proof. (1) For $A \subseteq X$, from Theorem 3.7, wsC(A) is a $w$-semiclosed set. Hence, $w I(w C(A)) \subseteq w I(w C(w s C(A))) \subseteq w s C(A)$.

(2) It is similar to the proof of (1).

Finally, we characterize $W$-semicontinuity in $w$-spaces in terms of the operators $w C, w I, w s C$ and $w s I$ as the following theorem:

Theorem 17. Let $f:\left(X, w_{X}\right) \rightarrow\left(Y, w_{Y}\right)$ be a function on $w$-spaces $X$ and $Y$. Then the following statements are equivalent:

(1) $f$ is $W$-semicontinuous.

(2) $f^{-1}(V) \subseteq w C\left(w I\left(f^{-1}(V)\right)\right)$ for each $w$-open set $V$ in $Y$.

(3) $w I\left(w C\left(f^{-1}(F)\right)\right) \subseteq f^{-1}(F)$ for each $w$-closed set $F$ in $Y$.

(4) $f(w I(w C(A))) \subseteq w C(f(A))$ for $A \subseteq X$.

(5) $w I\left(w C\left(f^{-1}(B)\right)\right) \subseteq f^{-1}(w C(B))$ for $B \subseteq Y$.

(6) $f^{-1}(w I(B)) \subseteq w C\left(w I\left(f^{-1}(B)\right)\right)$ for $B \subseteq Y$.

Proof. (1) $\Leftrightarrow$ (2) It follows from Theorem 3.11 and definition of $w$-semiopen sets.

(1) $\Leftrightarrow(3)$ It follows from Theorem 3.11 and Lemma 3.2.

$(3) \Rightarrow(4)$ Let $A \subseteq X$. Then from Theorem 3.11 and Lemma 3.12, it follows $w I(w C(A)) \subseteq w s C(A) \subseteq f^{-1}(w C(f(A)))$. Hence, $f(w I(w C(A))) \subseteq$ $w C(f(A))$.

(4) $\Rightarrow(5)$ Obvious.

$(5) \Rightarrow(6)$ From (5) and Theorem 2.3, it follows

$$
\begin{aligned}
f^{-1}(w I(B)) & =f^{-1}(Y-w C(Y-B)) \\
& =X-\left(f^{-1}(w C(Y-B))\right) \\
& \subseteq X-w I\left(w C\left(f^{-1}(Y-B)\right)\right) \\
& =w C\left(w I\left(f^{-1}(B)\right)\right) .
\end{aligned}
$$

Hence, $f^{-1}(w I(B)) \subseteq w C\left(w I\left(f^{-1}(B)\right)\right)$.

$(6) \Rightarrow(1)$ Let $V$ be a $w$-open set in $Y$. Then by (6) and Theorem 2.3 , we have $f^{-1}(V)=f^{-1}(w I(V)) \subseteq w C\left(w I\left(f^{-1}(V)\right)\right)$. This implies that $f^{-1}(V)$ is a $w$-semiopen set. Hence, by (2) of Theorem 3.11, $f$ is $W$-semicontinuous. 


\section{References}

[1] P. Bhattacharyya and B. K. Lahiri, Semi-generalized closed sets in topology, Indian J. Math., 29, No. 3 (1987), 375-382.

[2] Á. Csázár, Generalized topology, generalized continuity, Acta Math. Hungar., 96 (2002), 351-357.

[3] J. Dontchev and H. Maki, On sg-closed sets and semi- $\lambda$-closed sets, $Q \mathscr{G} A$ in General Topology, 15 (1997), 259-266.

[4] D. C. Kent and W. K. Min, Neighborhood spaces, International Journal of Mathematics and Mathematical Sciences, 32, No. 7 (2002), 387-399.

[5] N. Levine, Generalized closed sets in topology, Rend. Cir. Mat. Palermo, 19 (1970), 89-96.

[6] N. Levine, Semi-open sets and semi-continuity in topological spaces, Ams. Math. Monthly, 70 (1963), 36-41.

[7] H. Maki, J. Umehara and T. Noiri, Every topological space is pre- $T_{\frac{1}{2}}$, Mem. Fac. Sci. Kochi Univ. Ser. A, 17 (1996), 33-42.

[8] H. Maki, R. Devi and K. Balachandran, Associated topologies of generalized $\alpha$-closed sets and $\alpha$-generalized closed sets, Mem. Fac. Sci. Kochi Univ. Ser. A, 15 (1994), 51-63.

[9] H. Maki, R. Devi and K. Balachandran, Generalized $\alpha$-closed maps and $\alpha$-generalized closed maps, Indian J. pure appl. Math., 29, No. 1 (1998), 37-49.

[10] H. Maki, On generalizing semi-open and preopen sets, Report for Meeting on Topological Spaces Theory and its Applications, August 1996, Yatsushiro College of Technology, 1318.

[11] A. S. Mashhour, M. E. Abd El-Monsef and S. N. El-Deeb, On precontinuous and weak precontinuous mappings, Proc. Math. and Phys. Soc. Egypt, 53 (1982), 47-53.

[12] W. K. Min, Some Results on Generalized Topological Spaces and Generalized Systems, Acta Math. Hungar., 108, No-s: 1-2 (2005), 171-181.

[13] W. K. Min, On Weak Neighborhood Systems and Spaces, Acta Math. Hungar., 121, No. 3 (2008), 283-292.

[14] W. K. Min and Y. K. Kim, On Weak Structures and w-spaces, Far East Journal of Mathematical Sciences, 97, No. 5 (2015), 549-561.

[15] O. Njastad, On some classes of nearly open sets, Pacific Journal of Mathematics, 15, No. 3 (1964), 961-970.

[16] F. Siwiec, On Defining a Space by a Weak Base, Pacific Journal of Mathematics, 52, No. 1 (1974), 351-357. 